\title{
Preface: the 2013 international workshop on optical wave and waveguide theory and numerical modelling
}

\author{
Manfred Hammer • Milan Maksimović • \\ Mustafa Akin Sefunc • Remco Stoffer
}

Received: 15 January 2014 / Accepted: 16 January 2014 / Published online: 2 February 2014

C Springer Science+Business Media New York 2014

The XXI International Workshop on Optical Wave \& Waveguide Theory and Numerical Modelling (OWTNM 2013) was held in Enschede, the Netherlands, on the campus of the University of Twente, April 19 and 20, 2013. The annual workshop has been, since 1992, a forum for enthusiastic scientists in the field of integrated optics to exchange ideas, and to discuss problems, related to optical theory, computational modelling, and novel device concepts.

Integrated optics must nowadays be seen in an evolutionary context of related fields such as photonic nanostructures, metamaterials, and plasmonics, not to speak of the established areas of optical micro-resonators or photonic crystals. With the continuously increasing available computing power, the emphasis of simulation techniques shifts towards the modelling of larger or more complex systems, towards higher dimensionality, or towards more rigorous simulations. The field has thus seen quite some broadening, when compared to its origins. Respective suggestions from the 2012 meeting of the OWTNM technical committee led to a slight modification of the title of the workshop, where the traditional term waveguide gave way to the broader wave. Some emphasis on the traditional problems of integrated optics remains with the waveguide-subscript. This change might be continued or reversed for future editions of the OWTNM; the slight shift of emphasis, however, is well perceptible in the technical program of the workshop.

\footnotetext{
M. Hammer $(\varangle) \cdot$ M. A. Sefunc

MESA+ Institute for Nanotechnology, University of Twente, Enschede, The Netherlands e-mail:m.hammer@utwente.nl
}

M. A. Sefunc

e-mail:m.a.sefunc@utwente.nl

M. Maksimović

Focal Optical Systems, Enschede, The Netherlands

e-mail: milan.maksimovic@focal.nl

R. Stoffer

PhoeniX Software, Enschede, The Netherlands

e-mail: remco.stoffer@phoenixbv.com 
The OWTNM 2013 encompassed 56 scientific contributions, including 9 invited talks, distributed over one poster session and 8 oral sessions, on methods and algorithms I and II, active structures, functional devices, nanophotonics, metamaterials and nanostructures, physical phenomena, and plasmonics. This $12 \mathrm{~h}$ burden of formal scientific exchange was balanced by about $4 \mathrm{~h}$ of coffee and lunch breaks, and by the traditional workshop dinner on the first evening. We were happy to welcome about 65 participants (14\% female, $28 \%$ (PhD-) student registrations, $10 \%$ with commercial affiliation) from four continents.

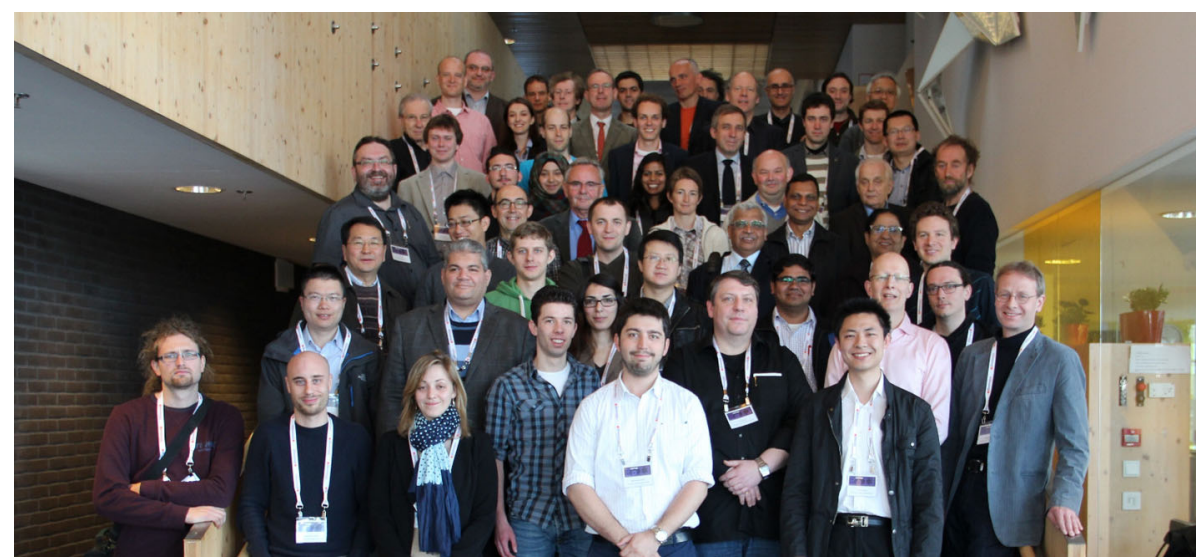

Participants of the XXI International Workshop on Optical Wave \& Waveguide Theory and Numerical Modelling, Enschede, The Netherlands, April 19-20, 2013

The organizers were grateful for the interest and the financial support of the following companies and institutions: PhoeniX Software, The Netherlands, MESA ${ }^{+}$Institute for Nanotechnology, University of Twente, JCMwave GmbH, Germany, Optiwave Systems Inc., Canada, IEEE Photonics Society, Benelux Chapter, Lumerical Solutions Inc., Canada, Focal Machine Vision \& Optical Systems, The Netherlands, and LioniX BV, The Netherlands. Their delegates were present at the workshop; the companies and institutions had posters on display in a separate section during the poster session.

Adhering to a tradition of the OWTNM series, this special issue of Optical and Quantum Electronics has been organized on the occasion of the workshop. Note that the issue includes, beside full papers that relate to actual oral or poster presentations at the workshop, also other papers authored by participants or by other interested scientists, that fall within the OWTNM scope.

The advice of the following members of the OWTNM technical committee is highly appreciated: Trevor Benson (University of Nottingham, UK), Jiří Čtyroký (Institute of Electronics and Photonics, Czech Republic), Manfred Hammer (University of Twente, The Netherlands), Andrei V. Lavrinenko (COM-DTU, Lyngby Kgs., Denmark), John Love (Australian National University, Canberra, Australia), Andrea Melloni (DEI-Politecnico di Milano, Italy), Olivier Parriaux (Lyon University at Saint Etienne, France), Reinhold Pregla (FernUniversität Hagen, Germany), Ivan Richter (Czech Technical University, Prague, Czech Republic), and Christoph Wächter (Fraunhofer IOF, Jena, Germany).

Special thanks go out to Hugo J.W.M. Hoekstra, Sonia García Blanco, and Lantian Chang (all University of Twente), of the local organizing committee, for their great contributions to the success of the workshop. 
We have enjoyed our task as guest editors of the present volume of Optical and Quantum Electronics. We thank all authors for submitting their work to this special issue. We would like to thank the reviewers for scrutinizing the submissions, and for their valuable comments that have contributed to the quality and clarity of the reported work. Finally our thanks go to Manikandan Jayaraman from the Springer editorial office, and to Editor-in-Chief of OQE, Trevor Benson, for their efficient and patient support.

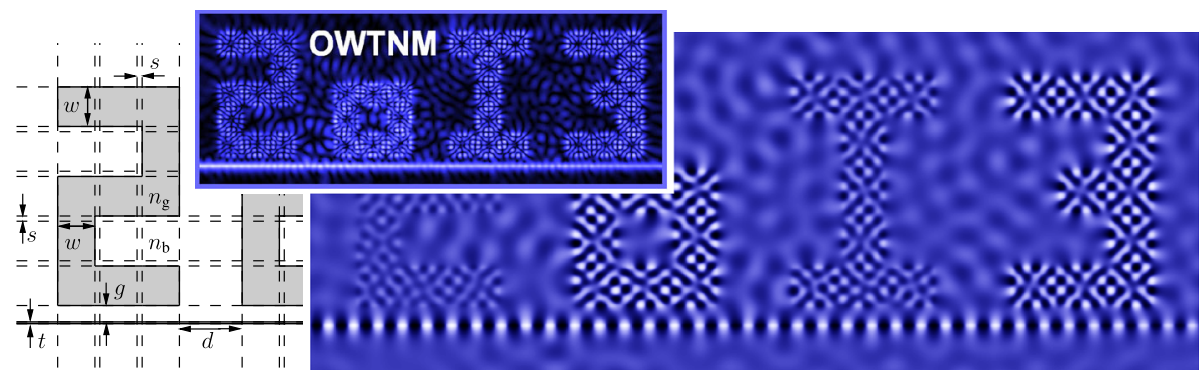

The OWTNM 2013 logo (central inset) and the underlying simulation: Rectangular arrangement of high index squares and spacers, excitation through the bottom slab channel, a 2-D configuration. All letters resonate with reasonably balanced intensity. Note that field maxima appear at different times within a period. Time snapshot of the principal electric component (TE) and field modulus (the logo). Refractive indices $n_{g}=3.4, n_{b}=1.45$, distances $w=1.451 \mu \mathrm{m}$, $s=0.192 \mu \mathrm{m}, d=2.44 \mu \mathrm{m}, t=0.073 \mu \mathrm{m}, \mathrm{g}=0.6 \mu \mathrm{m}$, vacuum wavelength $\lambda=1.55 \mu \mathrm{m}$. 